OPEN ACCESS

Edited by:

George Weinstock,

Jackson Laboratory for Genomic

Medicine, United States

Reviewed by:

Rafael Franco-Cendejas,

National Institute of Rehabilitation Luis

Guillermo Ibarra Ibarra, Mexico

Lingyun Guo,

Capital Medical University, China

${ }^{*}$ Correspondence:

Min Xu

xumin3568@sina.com

Yonggang LuO

Luoyg_514@126.com

${ }^{t}$ These authors have contributed equally to this work and share first authorship

Specialty section:

This article was submitted to

Clinical Microbiology,

a section of the journal

Frontiers in Cellular and

Infection Microbiology

Received: 01 March 2021

Accepted: 11 August 2021

Published: 10 September 2021

Citation:

Shi Q, Zhang J, Wang J,

Du L, Shi Z, Xu M and

Luo Y (2021) Homologous

Escherichia coli Identified in Cerebrospinal Fluid and Bloodstream. Front. Cell. Infect. Microbiol. 11:674235. doi: 10.3389/fcimb.2021.674235

\section{Homologous Escherichia coli Identified in Cerebrospinal Fluid and Bloodstream}

\author{
Qingmiao Shi ${ }^{1,2+}$, Jun Zhang ${ }^{3+}$, Jinghui Wang ${ }^{4}$, Lijuan $\mathrm{Du}^{4}$, Zhaoyang Shi ${ }^{4}$, \\ Min $\mathrm{Xu}^{5 *}$ and Yonggang Luo ${ }^{4 *}$
}

\begin{abstract}
1 Gene Hospital of Henan Province, The First Affiliated Hospital of Zhengzhou University, Zhengzhou, China, ${ }^{2}$ Department of Infectious Diseases, The First Affiliated Hospital of Zhengzhou University, Zhengzhou, China, ${ }^{3}$ Department of Pharmacy, The First Affiliated Hospital of Zhengzhou University, Zhengzhou, China, ${ }^{4}$ Department of Intensive Care Unit, The First Affiliated Hospital of Zhengzhou University, Zhengzhou, China, ${ }^{5}$ Department of Clinical Laboratory, The First Affiliated Hospital of Zhengzhou University, Zhengzhou, China
\end{abstract}

Background: Escherichia coli is an opportunistic bacterium that causes a wide range of diseases, such as bloodstream infection and central nervous system infection. The traditional culture-based method to detect $E$. coli usually takes more than 2 days. The object of this study is to explore the value of metagenomic next-generation sequencing (mNGS) in identifying E. coli from human cerebrospinal fluid. In addition, we investigated the infection source of $E$. coli through whole genome sequencing and phylogenetic analysis.

Methods: We combined a clinical example to analyze the function of mNGS in pathogen detection from cerebrospinal fluid. NextSeq 550Dx platform was applied for mNGS. Next, whole genome sequencing was performed to obtain the genomic characterization of E. coli. Furthermore, we screened 20 E. coli strains from the National Center for Biotechnology Information and conducted a phylogenetic analysis.

Results: A middle-aged patient who attended our hospital was diagnosed with craniopharyngioma and received surgery. The patient had recurrent fever and persistent lethargy after surgery. Cerebrospinal fluid culture firstly failed to grow the bacteria. Next the cerebrospinal fluid sample was detected by mNGS and the sequence readings of $E$. coli were identified. Later, $E$. coli was reported via the second cerebrospinal fluid culture, certifying the result of mNGS. Moreover, we also cultured carbapenemresistant $E$. coli from the patient's bloodstream. Through whole genome sequencing and phylogenetic analysis, we found that the $E$. coli isolated from cerebrospinal fluid and the bloodstream was 100\% homologous, indicating the E. coli central nervous system infection was originated from the bloodstream.

Conclusion: Metagenomic next-generation sequencing is a valuable tool to identify the pathogens from cerebrospinal fluid, and seeking the infection source is of great significance in clinical diagnosis and treatment. Furthermore, carbapenem-resistant $E$. coli is a serious problem as the cause of bloodstream infection and central nervous 
system infection, and effective and adequate measures to prevent and control the present circumstance are urgent.

Keywords: Escherichia coli, whole genome sequencing, phylogenetic analysis, homology, craniopharyngioma, metagenomic next-generation sequencing

\section{INTRODUCTION}

Escherichia coli is a common gram-negative bacterium that can be a harmless inhabitant of the gastrointestinal tract in humans (Croxen et al., 2013). These symbiotic E. coli strains seldom contribute to illness. However, once the individual's immune dysfunction or gut barrier is destroyed, it is also pathogenic and can result in many diseases, including diarrheal and extraintestinal diseases such as bloodstream infection, urinary tract infection, and central nervous system (CNS) infection (Kaper et al., 2004). The traditional diagnosis for pathogenic $E$. coli has depended on culture-based techniques (Peña-Gonzalez et al., 2019), but it usually takes more than 2 days. With the development of molecular diagnostic tools such as metagenomic next-generation sequencing (mNGS), it provides a handy sensitivity method to rapidly detect the pathogens ( $\mathrm{Li}$ et al., 2021).

Craniopharyngioma is a rare benign epithelial tumor of the CNS derived from residual cells of Rathke's pouch, which includes two different histopathological type, adamantinomatous and papillary craniopharyngioma (Müller, 2014). It constitutes about 2-5\% of primary intracranial tumors with an overall morbidity of $0.5-2$ cases per million (Erfurth et al., 2013). Approximately 60\% of all cases emerge in children, and the second peak of onset is the age of 40 to
44 years old. The invasion, adhesion, and compression of key structures around the sellar region lead to high mortality of craniopharyngioma even while receiving treatment (Karavitaki et al., 2005; Zoicas and Schöfl, 2012). The most common postoperative complications are involved in neuroendocrine dysfunction, visual deficits, and hypothalamic metabolic disorder. Although intracranial infection is seldom reported after surgery, it's a serious problem and shouldn't be ignored by clinicians.

Our study herein reported a febrile and lethargic patient who had undergone excision surgery of adamantinomatous craniopharyngioma. We identified $E$. coli sequence readings from the patient's cerebrospinal fluid (CSF) by mNGS when the traditional CSF culture first failed to grow a pathogen. Furthermore, we confirmed that the E. coli intracranial infection was sourced from bloodstream through whole genome sequencing and phylogenetic analysis, thus providing a modest recommendation for clinical management (Figure 1).

\section{MATERIALS AND METHODS}

\section{Patient and Samples}

Our study involved a middle-aged patient who was diagnosed with craniopharyngioma and received surgery at our hospital.

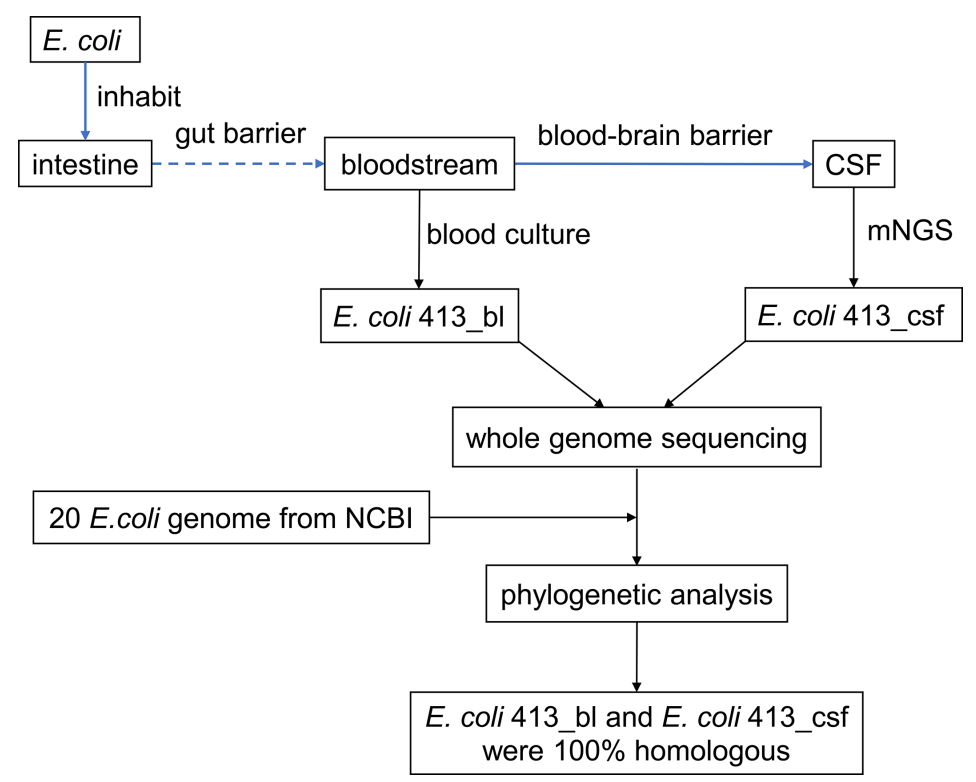

FIGURE 1 | Study design and flow diagram. CSF, cerebrospinal fluid; mNGS, metagenomic next-generation sequencing; NCBI, National Center for Biotechnology Information. 
Because of recurrent fever and persistent lethargy after surgery, the patient's CSF sample was collected after lumbar puncture by an experienced intensive care unit doctor, and the E. coli sequence readings were detected from CSF by mNGS. In addition, two suits of blood cultures were taken for the patient; one suit was from peripheral vein and another from central venous line. Each set included an aerobic bottle and an anaerobic bottle. And all the four bottles cultured carbapenem-resistant $E$. coli. The antimicrobial susceptibility test was detected by Kirby-Bauer and broth dilution method.

\section{Metagenomic Next-Generation Sequencing}

After CSF sample preprocessing, the microbial genomic DNA extraction was performed by TIANamp Micro DNA Kit (TIANGEN Biotech, Beijing) according to the manufacturer's instruction. Next, library construction was carried out, including genomic DNA fragmented, end-repair, 3' adenylated, adapters ligation, PCR amplifying, and purified products. The qualified single-stranded circular DNA library was sequenced on NextSeq 550 Dx platform (illumina, San Diego, USA) with a single-end 75bp sequencing strategy. We obtained the sequencing depth (Supplementary 1) using the complete genome of Escherichia coli str. K-12 substr. MG1655 (NCBI Reference Sequence: NC_000913.3) as genome reference sequence. Finally, we conducted a bioinformatics analysis and determined the mNGSbased pathogenic microorganism. The result revealed that mNGS identified 32,296 sequence readings of E. coli in the CSF sample.

\section{Whole Genome Sequencing}

Next, the E. coli strains isolated from CSF and the bloodstream were sequenced by whole genome sequencing, respectively. Firstly, the genomic DNA of E. coli was extracted by using MagPure DNA Kit (Cat. No. MD5118-05F, Magen, China). Secondly, we determined the concentration and purity of DNA through Qubit fluorometer and Nanodrop 2000 spectrophotometer (Thermo Fisher Scientific, Carlsbad, CA, USA). Thirdly, library construction and whole genome sequencing was performed on the MGISEQ-2000 platform at BGI (Shenzhen, China). The antibiotic-resistant genes were annotated using CARD (Comprehensive Antibiotic Resistance Database Version 3.1.2).

\section{Screening of E. coli Strains}

Twenty E. coli strains with genome information were screened out from the National Center for Biotechnology Information (https://www.ncbi.nlm.nih.gov). The 20 E. coli strains meet the following criteria: (1) the hosts were homo sapiens or human; (2) the isolation source was blood, CSF, or feces; (3) the collection date was in the last 10 years, except that the strain CE10 isolated from CSF was collected in the 1970s. The genome assembly and annotation data of the $20 \mathrm{E}$. coli strains were downloaded from Genbank (https://ftp.ncbi.nlm.nih.gov/ genomes/genbank/) for a genomic phylogenetic analysis.

\section{Phylogenetic Analysis}

Roary, a pipeline that structures large-scale pan genomes at a high speed (Page et al., 2015), was used to construct the pan genome and core genome of $22 \mathrm{E}$. coli strains. Next, we adopted
ClonalFrameML to analyze and detect recombination in bacterial whole genomes (Didelot and Wilson, 2015). Single nucleotide polymorphisms distance matrix with snp-dists was calculated. Furthermore, we performed phylogenetic analysis and a phylogenetic tree of $22 \mathrm{E}$. coli strains with branch lengths was reconstructed to display the evolutionary relationships.

\section{RESULTS}

\section{Case Presentation}

A middle-aged patient who complained of blurred vision with headache for three years was admitted to the neurosurgery department on July 23, 2020. The patient had a two-year history of type II diabetes and received oral hypoglycemic drugs regularly. Laboratory tests showed the fasting plasma glucose of $6.76 \mathrm{mmol} / \mathrm{L}$, with glycated hemoglobin of $7.10 \%$. Other tests were within normal range including blood routine examination, blood biochemistry, coagulation function, and thyroid function. Head magnetic resonance imaging scanning indicated a suprasellar space-occupying lesion (Figure 2A), which was suspected as craniopharyngioma. After a thorough evaluation, on August 3, the patient was administered the resection of the lesion and repair of CSF rhinorrhea by endoscopic endonasal transsphenoidal approach, which was confirmed to be adamantinomatous craniopharyngioma by histopathological examination of a surgical specimen afterwards (Figure 2B).

Despite using cefotiam (1g, q12h) as prophylactic treatment for surgery, the patient developed fever and lethargy the following day post-surgery. Reexamination of blood routine reported raised white blood cell of $13.49 \times 10^{9} / \mathrm{L}$ (reference range $3.9-9.5 \times 10^{9} / \mathrm{L}$ ) and percentage of neutrophils of $92.1 \%$ (reference range 40-75\%). The hemoglobin content $106.0 \mathrm{~g} / \mathrm{L}$ (reference range 115-150 g/L) and percentage of lymphocyte 6.2\% (reference range 20-50\%) were reduced. Lumbar puncture was performed and CSF tests revealed elevated glucose 15.29 $\mathrm{mmol} / \mathrm{L}$ (reference range $2.5-4.4 \mathrm{mmol} / \mathrm{L}$ ), total protein 5982.00 $\mathrm{mg} / \mathrm{L}$ (reference range 100-300 mg/L), and count of white blood cells $192.00 \times 10^{6} / \mathrm{L}$. Continuous monitoring of the CSF indicated no sign of CNS infection. Bacterial culture and gram stains of CSF reported negative results, respectively. The patient was given empiric anti-infective treatment with latamoxef sodium $(1.5 \mathrm{~g}$, $\mathrm{q} 12 \mathrm{~h}$ ) and vancomycin (1g, q12h) intravenously. Six days later, the patient's condition was improved.

On August 18, the patient occurred CSF rhinorrhea. Repair surgery of CSF rhinorrhea was performed by endoscopic endonasal transsphenoidal approach immediately. However, the patient developed fever and lethargy again the day after surgery, which had high possibility of intracranial infection considering that the characteristics of CSF was bloody and cloudy. Vancomycin (1g, $\mathrm{q} 12 \mathrm{~h})$ and cefoperazone-sulbactam (3g, q12h) were given for antiinfection intravenously, but the fever didn't reduce. To make matters worse, the patient emerged in diabetic ketoacidosis and was transmitted to intensive care unit urgently. CSF was bloody and cloudy with $0.77 \mathrm{mmol} / \mathrm{L}$ of glucose, and the glucose ratio was 0.06 . The count of white blood cells in CSF was increased to $187385.00 \times 10^{6} /$ 

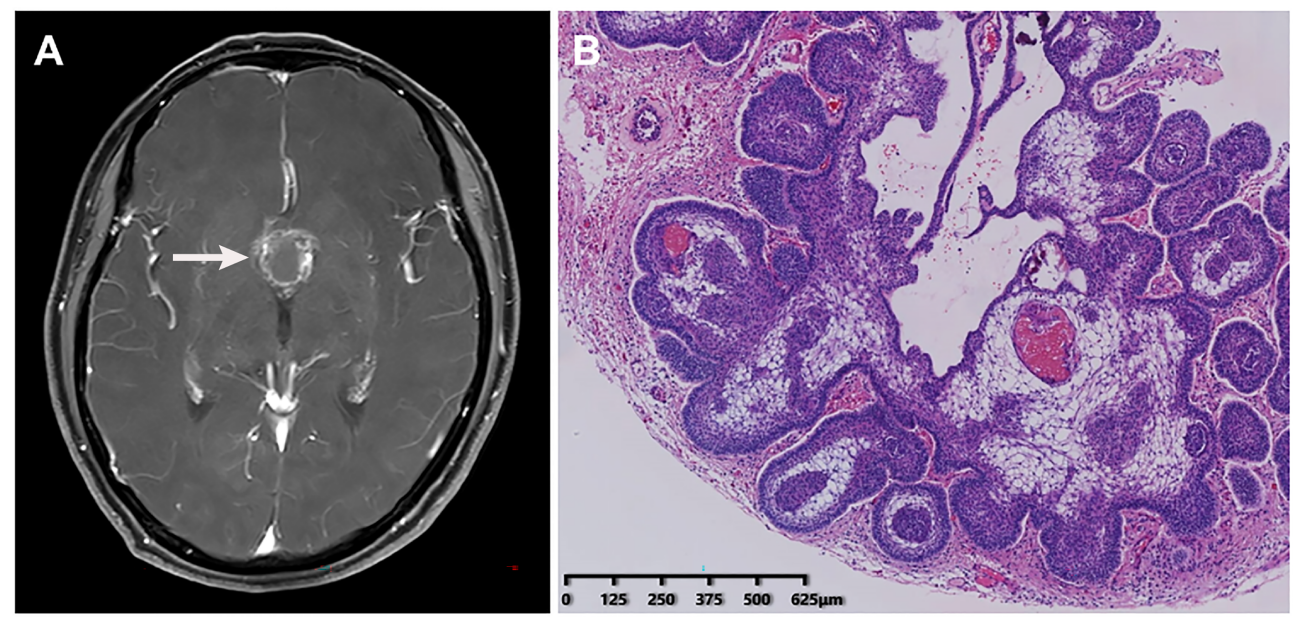

FIGURE 2 | Radiological imaging and histopathological examination of the patient. (A) Head magnetic resonance imaging showed a suprasellar space-occupying lesion. (B) Histopathological examination of a surgical specimen indicated adamantinomatous craniopharyngioma.

L and the total protein was $4098.5 \mathrm{mg} / \mathrm{L}$. But the bacterial culture of CSF was negative as before. In order to further seek the pathogen, the CSF was tested by mNGS and 32,296 sequence readings of E. coli was identified in CSF. Next, E. coli was also reported via the second cerebrospinal fluid culture, certifying the result of mNGS. After consultation with the pharmacy department, meropenem (2g, q8h), tigecycline (100mg, q12h), and linezolid (0.6g, q12h) were applied intravenously for this patient. To our surprise, carbapenem-resistant $E$. coli was cultured in the patient's bloodstream later. The minimum inhibitory concentration (MIC) was measured by broth dilution method. The antimicrobial susceptibility test showed a multi-drug resistance of $E$. coli to doxycycline (MIC $\geq 16 \mathrm{ug} / \mathrm{ml}$ ), piperacillintazobactam (MIC $\geq 128 \mathrm{ug} / \mathrm{ml}$ ), cefoperazone/sulbactam (MIC $\geq 64$ $\mathrm{ug} / \mathrm{ml}$ ), ceftazidime (MIC $\geq 64 \mathrm{ug} / \mathrm{ml}$ ), cefepime (MIC $\geq 32 \mathrm{ug} / \mathrm{ml}$ ), aztreonam (MIC $\geq 64 \mathrm{ug} / \mathrm{ml})$, meropenem (MIC $\geq 16 \mathrm{ug} / \mathrm{ml})$, imipenem (MIC $\geq 16 \mathrm{ug} / \mathrm{ml}$ ), levofloxacin (MIC $\geq 8 \mathrm{ug} / \mathrm{ml}$ ), and so on.

Questioning the medical history of the patient, we came to know that the patient had symptoms of diarrhea after the surgery for craniopharyngioma. Thus, we inferred that the gut microbiota translocations occurred in the state of surgical stress. The E. coli residing in the intestine became opportunistic bacterium and translocated into the blood, resulting in bloodstream infection. Furthermore, the E. coli in the bloodstream moved through the blood-brain barrier and entered the CSF, causing CNS infection. To prove the conjecture, whole genome sequencing analysis of E. coli strains isolated from CSF sample and bloodstream sample were performed. After phylogenetic analysis, the results suggested that the E. coli from CSF was $100 \%$ homologous with the E. coli from the blood. It indicated that the intracranial $E$. coli infection of the patient was not caused by the invasive surgical procedure but originated from the bloodstream. So far, the cause of the patient's intracranial infection can be clarified.

According to combined drug susceptibility test, the anti-infective regimen was adjusted to intravenous administration of ceftazidimeavibactam (2.5g, q8h, ivgtt) and aztreonam (2g, q6h, ivgtt), along with intrathecal injection of polymyxin B (5WIU). Considering the neurotoxicity of polymyxin $\mathrm{B}$, its use was discontinued after seven times intrathecal injections. Through 20 days of anti-infection treatment, the fever of this patient was controlled and her condition was getting better. CSF was transparent with white blood cells count of $53 \times 10^{6} / \mathrm{L}$, glucose of $5.49 \mathrm{mmol} / \mathrm{L}$, and total protein of $1319.0 \mathrm{mg} / \mathrm{L}$. Next the antibiotics were discontinued, the patient remained in the hospital under observation for a week, and then was discharged. Two weeks later, we followed up with the family of the patient. Unfortunately, we learned that the patient has expired outside hospital due to the uncontrolled infection caused by newly developed and untreated CSF leak. The schematic of the patient's clinical course was showed in Figure 3.

\section{The E. coli Strains Identified in Our Study}

From the CSF sample, we detected 32,296 sequence readings of E. coli genome by mNGS and this strain E. coli was named 413_csf. From the blood culture sample, we identified carbapenem-resistant E. coli, which was named 413_bl. Through whole genome sequencing, it showed that the E. coli 413_bl and 413_csf were homologous. The antibiotics-resistant genes data of whole genome sequencing was presented in Supplementary 2 . In addition, the carbapenem resistance arose from $\beta$-lactam ring hydrolysis by dedicated carbapenems enzymes and the carbapenems come from ambler class B metallo- $\beta$-lactamases.

\section{Genomic Characteristics}

We filtered out $20 \mathrm{E}$. coli strains with whole genome sequencing information from the National Center for Biotechnology Information. The genomic characteristics of $20 \mathrm{E}$. coli strains were presented in Table 1. Fourteen E. coli strains were isolated from the blood of homo sapiens; one strain was from CSF and five strains were from feces. The genome size and protein-coding sequences for $20 \mathrm{E}$. coli strains ranged from $4.82 \mathrm{Mb}$ to $5.71 \mathrm{Mb}$ 


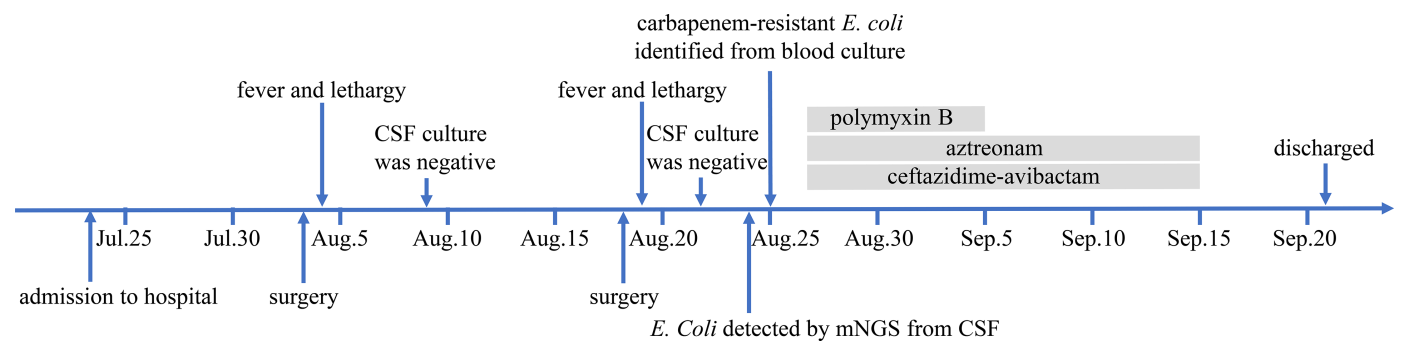

FIGURE 3 | The schematic of the patient's clinical course. CSF, cerebrospinal fluid; mNGS, metagenomic next-generation sequencing.

and 4006 to 5179 genes. The $\mathrm{G}+\mathrm{C}$ contents of the 20 E. coli genome were between $50.39 \%$ and $51.21 \%$.

\section{Genomic Recombination Analysis}

We used Roary to create a pan-genome and core-genome of 22 strains and got 2654 core genes (99\% $\leq$ strains $\leq 100 \%)$ and 14,854 pan genes $(0 \% \leq$ strains $\leq 100 \%)$. Then we identified recombination with ClonalFrameML and got a pairwise single nucleotide polymorphisms distance matrix with snp-dists (Supplementary 3) from a FASTA sequence alignment after we masked the recombination regions. We found that the snp-dists between E. coli 413_bl and 413_csf was 0 , which provided evidence that the two E. coli strains were highly homologous.

\section{Phylogenetic Analysis for E. coli}

We performed phylogenetic analysis for E. coli and conducted a phylogenetic tree based on the whole genome sequencing data of 22 E. coli strains (Figure 4). It showed that E. coli 413 bl and 413_csf exist in the same clade with another five strains, including E. coli GZEC065, SJ7, 2EC1, NCCP15955, RM-103-MS, indicating that E. coli 413_bl and 413_csf have close genetic relationship with them. Combining the result that the snp-dists between E. coli 413_bl and 413_csf was 0, which further confirmed the $100 \%$ homologousness of them. E. coli GZEC065 was isolated from the blood of homo sapiens. E. coli SJ7, 2EC1, NCCP15955, and RM103-MS were isolated from the feces of homo sapiens. Thus, we inferred that the E. coli 413_bl in our case may be from the intestine, and the E. coli 413_bl in the bloodstream entered the cerebrospinal fluid through the blood-brain barrier, causing intracranial infection. The only strain isolated from CSF, E. coli CE10, had a relatively far connection with E. coli 413_csf. We infer that that's because E. coli CE10 was collected from newborn with bacterial meningitis in 1970 s, which is ancient and not sourced from the intestine (Lu et al., 2011).

\section{DISCUSSION}

In this study, we identified E. coli from cerebrospinal fluid by mNGS under circumstance of initial traditional culture-negative, to assist

TABLE 1 | Genome statistical information of the 20 Escherichia coli strains selected from NCBI.

\begin{tabular}{|c|c|c|c|c|c|c|c|c|}
\hline Strain & Assembly.NO & Genome Size (Mb) & CDS & $\mathrm{G}+\mathrm{C}(\mathrm{mol} \%)$ & Country & Isolation source & Host disease & Collection date \\
\hline $188 \mathrm{~B}$ & GCA_014898615.1 & 5.13381 & 4096 & 51.21 & Turkey & Blood & Sepsis & 2018 \\
\hline 188 & GCA_014898635.1 & 5.1333 & 4006 & 51.11 & Turkey & Blood & Sepsis & 2018 \\
\hline $2 \mathrm{D}$ & GCA_013425955.1 & 5.3633 & 4930 & 50.61 & China & Blood & Bloodstream infection & 2019 \\
\hline 190693 & GCA_011022275.1 & 5.15195 & 4714 & 50.60 & UK & Blood & Bacteremia & 2016 \\
\hline p11A & GCA_011330935.1 & 5.54584 & 5166 & 50.76 & USA & Blood & Bacteremia & 2016 \\
\hline $\mathrm{p} 4 \mathrm{~A}$ & GCA_011331215.1 & 5.2555 & 4861 & 50.70 & USA & Blood & Bacteremia & 2016 \\
\hline GZEC065 & GCA_010092405.1 & 5.10792 & 4610 & 50.75 & China & Blood & NA & 2017 \\
\hline 69 & GCA_007107705.1 & 5.41794 & 5028 & 50.71 & USA & Blood & Bloodstream infection & 2014 \\
\hline 95 & GCA_007107905.1 & 5.06629 & 4662 & 50.72 & USA & Blood & Bloodstream infection & 2013 \\
\hline 131 & GCA_007108085.1 & 5.21412 & 4804 & 50.79 & USA & Blood & Bloodstream infection & 2012 \\
\hline 963 & GCA_007108305.1 & 5.25381 & 4756 & 50.70 & USA & Blood & Bloodstream infection & 2012 \\
\hline strain Z247 & GCA_002142715.1 & 5.40007 & 4972 & 50.39 & China & Blood & Bloodstream infection & 2015 \\
\hline Z1002 & GCA_002142675.1 & 5.62852 & 5152 & 50.52 & China & Blood & Bloodstream infection & 2014 \\
\hline SF-468 & GCA_001280345.1 & 5.35864 & 5012 & 50.61 & USA & Blood & Bloodstream infection & 2010 \\
\hline CE10 & GCA_000227625.1 & 5.37873 & 4911 & 50.58 & USA & CSF & Neonatal meningitis & 1970 \\
\hline RM-103-MS & GCA_014217095.1 & 5.70703 & 4353 & 50.50 & Bangladesh & Feces & Healthy individual & 2017 \\
\hline E686 & GCA_011045295.1 & 5.16231 & 4516 & 50.60 & Korea & Feces & NA & 2019 \\
\hline 2EC1 & GCA_009905115.1 & 4.86859 & 4383 & 50.90 & Singapore & Feces & Healthy individual & 2019 \\
\hline SJ7 & GCA_008761535.3 & 5.57204 & 5179 & 50.66 & USA & Feces & Bloody diarrhea & 2015 \\
\hline NCCP 15955 & GCA_009873375.1 & 4.82062 & 4266 & 50.60 & China & Feces & Diarrhea & 2014 \\
\hline
\end{tabular}

CDS, coding sequences; CSF, cerebrospinal fluid; NA, not available. 


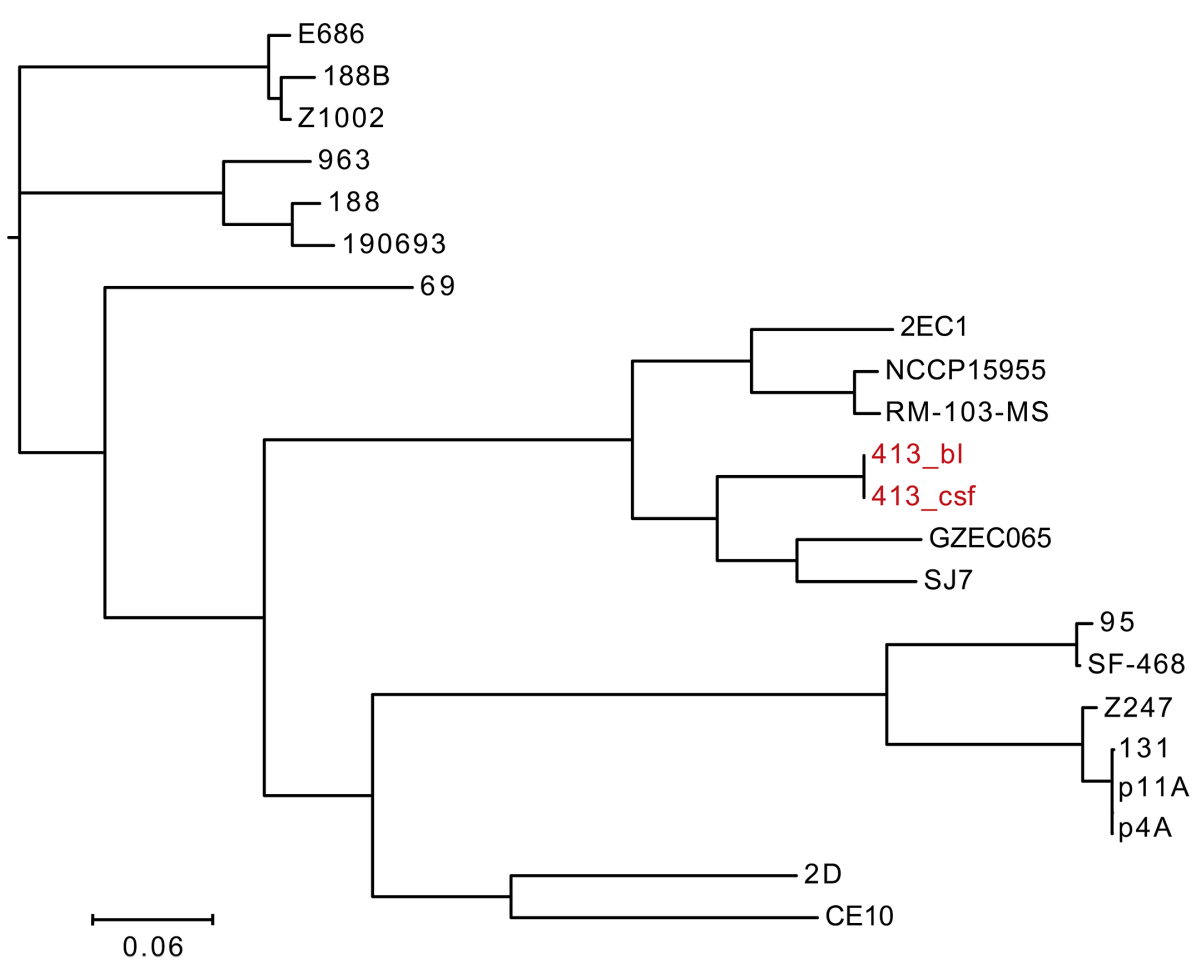

FIGURE 4 | Phylogenetic tree constructed using whole genomes of 22 E. coli strains, including E. coli 413_bl strains isolated from bloodstream, E. coli 413_csf isolated from cerebrospinal fluid, and $20 \mathrm{E}$. coli strains screened from the National Center for Biotechnology Information.

the clinicians in detecting infectious pathogen rapidly. And the second cerebrospinal fluid culture reported E. coli, further indicating the precision of mNGS. It's reported that the positive rate of cerebrospinal fluid culture in meningitis is only between $5.4 \%$ and 24.3\% in developing countries like China (Li et al., 2014; Zhang et al., 2020). In addition, the prophylactic use of inappropriate broad-spectrum antibiotics reduces the sensitivity of pathogens detection by conventional culture, causing challenges in diagnosing the pathogens (Paul et al., 2010).

Accumulating evidence have proved that mNGS is an unbiased and comprehensive diagnostic strategy and is less disturbed by preliminary antibiotics exposure (Miao et al., 2018). A prospective multicenter study revealed that the positive rate of mNGS in cerebrospinal fluid infection was 57.0\% (Xing et al., 2020). Moreover, it's found that the patient-as in our study-with CSF WBC $>300 \times 10^{6} / \mathrm{L}$, CSF protein $>500 \mathrm{mg} / \mathrm{L}$, or glucose ratio $\leq 0.3$ had a notably higher mNGS detection rate (Miao et al., 2018). Another retrospective observational study focused on CSF samples from 99 pediatric bacterial meningitis patients found that mNGS appeared with higher sensitivity (68.7\%) than culture (55.6\%), and the top four detected pathogens were Streptococcus pneumoniae, Streptococcus Agalactiae, Staphylococcus aureus, and Escherichia coli (Guo et al., 2019). In a word, it demonstrated that mNGS had preferable diagnosis efficiency to culture in CNS infection.

Postoperative CNS infection remains a severe question requiring instant attention in patients undergoing neurosurgery. A previous research in America found that $0.8 \%$ (14/1587) patients after cranial operations were complicated with intracranial infection, and the morbidity of bacterial meningitis was $0.3 \%$ (4/1587). In addition, this research observed no associations between cerebrospinal fluid leakage, diabetes mellitus, and CNS infection (McClelland and Hall, 2007). However, a retrospective study in China revealed that the risk of meningitis after major craniotomy was increased by the presence of diabetes mellitus and lumbar drainage (Chen et al., 2014). In our study, the patient had undergone surgery of craniopharyngioma resection by endoscopic endonasal transsphenoidal approach, with type 2 diabetes, cerebrospinal fluid leakage, and lumbar cistern drainage. No research reported the postoperative CNS infection associated risk factors of neurosurgical endoscopic endonasal transsphenoidal approach. Thus, it's meaningful to further explore this topic to provide appropriate recommendations for clinicians around reducing the complications after neurosurgical procedures.

In addition to the identification of E. coli in the CSF, we also cultured E. coli in the bloodstream. To explore the homology of the two isolated E. coli strains, whole genome sequencing was performed. After phylogenetic analysis, we confirmed that the two E. coli strains posed $100 \%$ homologous-meaning they are the same strain, which helped clinicians inferring the source of $E$. coli in CSF. Because the patient occurred CNS infection postsurgery, we couldn't exclude that the patient was infected during the surgery if without evidence. However, according to the results of whole genome sequencing, we concluded that the $E$. coli in CSF was originated from the bloodstream. In a way, it provides a reliable basis for the clinicians to clarify the causation 
to the patient. And the E. coli in bloodstream was carbapenemresistant via drug susceptibility test, thus contributing to formulating a precise antibiotic treatment.

E. coli is a major pathogen that causes bloodstream infection. In Oxfordshire, UK, about half of $E$. coli bloodstream infections were associated with urinary tract infection and three quarters of bloodstream infection with $E$. coli were community-onset (Vihta et al., 2018). With the increase of antibiotic resistance, the scope of therapy choices is shrinking (Harris et al., 2018). Our study identified carbapenem-resistant E. coli from bloodstream infection in the hospital, which could be related to diarrhea. According to the statistics, carbapenem-resistant Enterobacteriaceae has been a major global public health problem (Xu et al., 2015). Patients with carbapenem-resistant Enterobacteriaceae infection usually have a history of prolonged exposure to health care facilities, which is consistent with long-term and diversified antibiotic consumption. Antibiotics available to treat carbapenem-resistant Enterobacteriaceae are limited, and combination therapy with multiple antibiotics has been shown to reduce mortality. In our study, anti-infective treatment was used for 20 days in combination with ceftazidime-avibactam and aztreonam, and the fever of the patient was relieved.

\section{LIMITATIONS}

One pivotal limitation of our study was a lack of pathogen identification of the patient's stool, meaning that our inferences about the relation of $E$. coli bloodstream infection and diarrhea need to be confirmed. Second, it's important to administer early postoperative enteral nutrition to maintain the gastrointestinal homeostasis and the stability of gut microbiota. Third, we should strengthen the management of cerebrospinal fluid leakage in patients, trying to avoid the occurrence of it.

\section{CONCLUSION}

In summary, our study demonstrated the usefulness of mNGS in improved identification of neurosurgical postoperative CNS infection. Actively tracing the infection source is of great significance to clinical diagnosis and treatment. In addition, carbapenem-resistant E. coli is a serious problem as the cause of bloodstream infection and CNS infection, and effective and adequate measures to prevent and control the present circumstance are urgent.

\section{REFERENCES}

Chen, C., Zhang, B., Yu, S., Sun, F., Ruan, Q., Zhang, W., et al. (2014). The Incidence and Risk Factors of Meningitis After Major Craniotomy in China: A Retrospective Cohort Study. PloS One 9 (7), e101961. doi: 10.1371/ journal.pone.0101961

Croxen, M., Law, R., Scholz, R., Keeney, K., Wlodarska, M., and Finlay, B. (2013). Recent Advances in Understanding Enteric Pathogenic Escherichia Coli. Clin. Microbiol. Rev. 26 (4), 822-880. doi: 10.1128/cmr.00022-13

\section{DATA AVAILABILITY STATEMENT}

The data that support the findings of this study have been deposited into CNGB Sequence Archive (CNSA) of China National GeneBank DataBase (CNGBdb). The names of the repository/repositories and accession number(s) can be found below: https://db.cngb.org/, CNP0002005.

\section{ETHICS STATEMENT}

The studies involving human participants were reviewed and approved by the ethical committee of the First Affiliated Hospital of Zhengzhou University (2019-KY-330). The patients/ participants provided their written informed consent to participate in this study.

\section{AUTHOR CONTRIBUTIONS}

YL and MX designed the study. QS collected the data and performed the data analyses. JZ provided medication guidance. JW, LD, and ZS involved in patient management and provided clinical information. MX performed laboratory investigations. QS and JZ wrote the original manuscript. YL and MX revised and supervised the paper. All authors contributed to the article and approved the submitted version.

\section{FUNDING}

This work was equally funded and supported by Chinese National Science and Technology Major Project 2018ZX10305410, Henan Province Science and Technology Research Project grant 192102310054 and 212102310321, Fund of the First Affiliated Hospital of Zhengzhou University 2018020136.

\section{SUPPLEMENTARY MATERIAL}

The Supplementary Material for this article can be found online at: https://www.frontiersin.org/articles/10.3389/fcimb.2021. 674235/full\#supplementary-material

Didelot, X., and Wilson, D. (2015). ClonalFrameML: Efficient Inference of Recombination in Whole Bacterial Genomes. PloS Comput. Biol. 11 (2), e1004041. doi: 10.1371/journal.pcbi.1004041

Erfurth, E., Holmer, H., and Fjalldal, S. (2013). Mortality and Morbidity in Adult Craniopharyngioma. Pituitary 16 (1), 46-55. doi: 10.1007/s11102-012-0428-2

Guo, L., Li, Y., Liu, L., Wu, H., Zhou, J., Zhang, Y., et al. (2019). Detection of Pediatric Bacterial Meningitis Pathogens From Cerebrospinal Fluid by NextGeneration Sequencing Technology. J. Infect. 78 (4), 323-337. doi: 10.1016/ j.jinf.2018.12.001 
Harris, P., Tambyah, P., Lye, D., Mo, Y., Lee, T., Yilmaz, M., et al. (2018). Effect of Piperacillin-Tazobactam vs Meropenem on 30-Day Mortality for Patients With E Coli or Klebsiella Pneumoniae Bloodstream Infection and Ceftriaxone Resistance: A Randomized Clinical Trial. JAMA 320 (10), 984-994. doi: 10.1001/jama.2018.12163

Kaper, J., Nataro, J., and Mobley, H. (2004). Pathogenic Escherichia Coli. Nat. Rev. Microbiol. 2 (2), 123-140. doi: 10.1038/nrmicro818

Karavitaki, N., Brufani, C., Warner, J., Adams, C., Richards, P., Ansorge, O., et al. (2005). Craniopharyngiomas in Children and Adults: Systematic Analysis of 121 Cases With Long-Term Follow-Up. Clin. Endocrinol. 62 (4), 397-409. doi: 10.1111/j.1365-2265.2005.02231.x

Li, N., Cai, Q., Miao, Q., Song, Z., Fang, Y., and Hu, B. (2021). High-Throughput Metagenomics for Identification of Pathogens in the Clinical Settings. Small. Methods 5 (1), 2000792. doi: 10.1002/smtd.202000792

Li, Y., Yin, Z., Shao, Z., Li, M., Liang, X., Sandhu, H., et al. (2014). Population-Based Surveillance for Bacterial Meningitis in China, September 2006-December 2009. Emerging. Infect. Dis. 20 (1), 61-69. doi: 10.3201/eid2001.120375

Lu, S., Zhang, X., Zhu, Y., Kim, K., Yang, J., and Jin, Q. (2011). Complete Genome Sequence of the Neonatal-Meningitis-Associated Escherichia Coli Strain CE10. J. Bacteriol. 193 (24), 7005. doi: 10.1128/jb.06284-11

McClelland, S., and Hall, W. (2007). Postoperative Central Nervous System Infection: Incidence and Associated Factors in 2111 Neurosurgical Procedures. Clin. Infect. Dis. 45 (1), 55-59. doi: 10.1086/518580

Miao, Q., Ma, Y., Wang, Q., Pan, J., Zhang, Y., Jin, W., et al. (2018). Microbiological Diagnostic Performance of Metagenomic Next-Generation Sequencing When Applied to Clinical Practice. Clin. Infect. Dis. 67, S231S240. doi: 10.1093/cid/ciy693

Müller, H. (2014). Craniopharyngioma. Endocrine. Rev. 35 (3), 513-543. doi: 10.1210/er.2013-1115

Page, A., Cummins, C., Hunt, M., Wong, V., Reuter, S., Holden, M., et al. (2015). Roary: Rapid Large-Scale Prokaryote Pan Genome Analysis. Bioinf. (Oxford. England). 31 (22), 3691-3693. doi: 10.1093/bioinformatics/btv421

Paul, M., Shani, V., Muchtar, E., Kariv, G., Robenshtok, E., and Leibovici, L. (2010). Systematic Review and Meta-Analysis of the Efficacy of Appropriate Empiric Antibiotic Therapy for Sepsis. Antimicrob. Agents Chemother. 54 (11), 4851-4863. doi: 10.1128/aac.00627-10

Peña-Gonzalez, A., Soto-Girón, M., Smith, S., Sistrunk, J., Montero, L., Páez, M., et al. (2019). Metagenomic Signatures of Gut Infections Caused by Different
Escherichia coli Pathotypes. Appl. Environ. Microbiol. 85 (24), e01820-19. doi: 10.1128/aem.01820-19

Vihta, K., Stoesser, N., Llewelyn, M., Quan, T., Davies, T., Fawcett, N., et al. (2018). Trends Over Time in Escherichia Coli Bloodstream Infections, Urinary Tract Infections, and Antibiotic Susceptibilities in Oxfordshire, U-2016: A Study of Electronic Health Records. Lancet Infect. Dis. 18 (10), 1138-1149. doi: 10.1016/ s1473-3099(18)30353-0

Xing, X., Zhang, J., Ma, Y., He, M., Yao, G., Wang, W., et al. (2020). Metagenomic Next-Generation Sequencing for Diagnosis of Infectious Encephalitis and Meningitis: A Large, Prospective Case Series of 213 Patients. Front. Cell. Infect. Microbiol. 10, 88. doi: 10.3389/fcimb.2020.00088

Xu, Y., Gu, B., Huang, M., Liu, H., Xu, T., Xia, W., et al. (2015). Epidemiology of Carbapenem Resistant Enterobacteriaceae (CRE) During 2000-2012 in Asia. J. Thoracic. Dis. 7 (3), 376-385. doi: 10.3978/j.issn.2072-1439.2014.12.33

Zhang, Y., Cui, P., Zhang, H., Wu, H., Ye, M., Zhu, Y., et al. (2020). Clinical Application and Evaluation of Metagenomic Next-Generation Sequencing in Suspected Adult Central Nervous System Infection. J. Trans. Med. 18 (1), 199. doi: 10.1186/s12967-020-02360-6

Zoicas, F., and Schöfl, C. (2012). Craniopharyngioma in Adults. Front. Endocrinol. 3, 46. doi: 10.3389/fendo.2012.00046

Conflict of Interest: The authors declare that the research was conducted in the absence of any commercial or financial relationships that could be construed as a potential conflict of interest.

Publisher's Note: All claims expressed in this article are solely those of the authors and do not necessarily represent those of their affiliated organizations, or those of the publisher, the editors and the reviewers. Any product that may be evaluated in this article, or claim that may be made by its manufacturer, is not guaranteed or endorsed by the publisher.

Copyright (c) 2021 Shi, Zhang, Wang, Du, Shi, Xu and Luo. This is an open-access article distributed under the terms of the Creative Commons Attribution License (CC BY). The use, distribution or reproduction in other forums is permitted, provided the original author(s) and the copyright owner(s) are credited and that the original publication in this journal is cited, in accordance with accepted academic practice. No use, distribution or reproduction is permitted which does not comply with these terms. 